\title{
Aproximación Psico Comunitario Ambiental al Problema de Calentamiento Global
}

\section{An Environmental Community Approach to the Problem of Global Warming}

\author{
Germán Rozas O. ${ }^{1}$
}

\begin{abstract}
Resumen
La Psicología Comunitaria tiene mucho que decir en temas ambientales, en este artículo se presenta el grave problema del Calentamiento Global, y se señala como este fenómeno tiene consecuencias en la población humana. Se revisan sus causas y se pone énfasis en como la misma sociedad humana provoca el calentamiento del planeta a través de comportamientos y estilos de vida que llevan incorporados un enfoque totalmente economicista del medio ambiente. Se revisan otros paradigmas los cuales en general proponen un cambio, en el sentido de generar mayor conciencia de que los seres humanos son parte del ecosistema y por lo mismo no corresponde entender la naturaleza como una bodega de víveres para satisfacer sus necesidades. Se propone algunas líneas de intervención de carácter comunitaria, que abordan aspectos conceptuales como así mismo dimensiones operativas.
\end{abstract}

Palabras Claves: Psicología comunitaria, Calentamiento Global, Ecología, Intervención.

\begin{abstract}
Community Psychology has much to say about environmental topics. This article presents the very serious problem of Global Warming pointing out the consequence this phenomenon has on the human population. Causes are revised and an emphasis is laid on how human society itself provokes the warming of the planet through behaviors and life styles which incorporate a completely economical approximation to the environment. Other paradigms are reviewed which, in general, propose possible changes through generating the awareness that human beings are part of the ecosystem and for this reason nature should not be understood as a warehouse for food. Other lines of intervention are offered, from a community perspective, which could be undertaken in conceptual as well as operative dimensions.
\end{abstract}

Keys Words: Psychology community, Global Warming, Ecology.

1 Académico, Departamento de Psicología, Universidad de Chile. grozas@uchile.cl. 


\section{1.- Calentamiento Global}

El tema del Calentamiento Global se ha transformado hoy día en un ámbito estratégico. Fuera de ser un problema en sí mismo es un sujeto de estudio que establece contacto con un conjunto de temáticas que involucran a las ciencias sociales estableciendo puntos sensibles en diversas dimensiones. Es un tema pivote, de ello no hay duda, que toma contacto desde la biología, la ecología hasta la psicología.

La causa principal de este problema es el efecto invernadero, el cual no es un fenómeno nuevo e incluso no es un hecho exclusivamente humano. El Efecto Invernadero es un proceso natural, que siempre ha existido y que forma parte de los ciclos normales del funcionamiento del planeta, sin embargo el tema no es tan simple, la sociedad humana en su dinámica de crecimiento, particularmente en sus mecanismos de industrialización ha estimulado el efecto invernadero llevándolo a niveles anormales.

Una parte del calor del sol entra al planeta traspasando las barreras que opone la atmósfera. Luego de cumplir su función, este calor se devuelve hacia la atmósfera, una parte todavía del mismo escapa hacia el exterior y la parte restante se dirige nuevamente hacia el planeta reproduciendo el mismo ciclo. Aquí ya actuó el efecto natural del efecto invernadero. El cual se constituye en una especie de membrana permeable que hace rebotar el calor redirigiéndolo otra vez hacia la tierra. Se conforma a partir de la evaporación del agua del mar como así mismo de gases propios de la tierra.

La actividad humana interviene en el proceso natural del efecto invernadero, reforzándolo y llevándolo a constituirse en una verdadera muralla atmosférica impidiendo el necesario escape de calor proveniente del sol. De manera que en este nuevo escenario el volumen de calor que ingresa no vuelve a salir en la cantidad requerida produciéndo- se una acumulación del mismo, y con ello elevando el promedio de la temperatura de todo el planeta.

Los gases con los cuales la sociedad humana contribuye principalmente al efecto invernadero son el Anhídrido Carbónico, el Metano y el Oxido Nitroso. El primero de ellos, también llamado Dióxido de Carbono (CO2), el más importante y participa en la conformación del efecto invernadero en un $70 \%$.

Estos gases son producidos por la actividad humana. Las ciudades con su movilidad a través de diferentes medios de locomoción arrojan a la atmósfera enormes volúmenes de $\mathrm{CO} 2$, sin embargo, más importante aún son las emisiones que provoca la producción industrial de todos los países desde cualquier parte del mundo.

No obstante, hay aquí un proceso combinado. Existe de modo natural un proceso que contrarresta el efecto invernadero y esta es la enorme capacidad de la biósfera de retener y absorber el gas carbónico. Específicamente toda la flora con su gran cantidad de bosques sobre el planeta absorben dichos gases. Pero, desafortunadamente la misma sociedad y su modernidad limitan seriamente la puesta en acción de este efecto positivo.

La modernidad en su exigencia de aumentar el nivel de vida de la población, las sociedades en su afán de consumir sin freno, empujan a todos los países, cual más o cual menos hacia la deforestación.

La deforestación es eliminar los bosques y la fauna para transformar esos territorios en zonas agrícolas y en zonas ganaderas o en espacios de localización de ciudades. La deforestación avanza a un ritmo de 17 millones de hectáreas al año, lo que constituye una superficie de mayor que toda Inglaterra, Gales e Irlanda del norte juntas. La deforestación esta afectando particularmente el amazonas, el gran pulmón de América Latina y del mundo (www.ecoportal.net, Octubre de 2003) 
Ahora, en relación a la emisión de gases de carbono a la atmósfera, sin duda la responsabilidad recae sobre la sociedad humana, sin embargo no todos los países del mundo pueden ser acusados al respecto. Todo lo contrario, son en esencia los países desarrollados a quienes principalmente se les puede achacar tal situación.
El noventa y cinco por ciento de las emisiones industriales de $\mathrm{CO} 2$ son producidas en el hemisferio norte, EE.UU. es el principal responsable de la emisión de los gases carbono, con un $25 \%$ del total de emisiones, dicho país fue el único que no firmó los acuerdos sobre la reducción de gases invernadero, en la cumbre de Kyoto, Japón realizada en Diciembre de 1997.

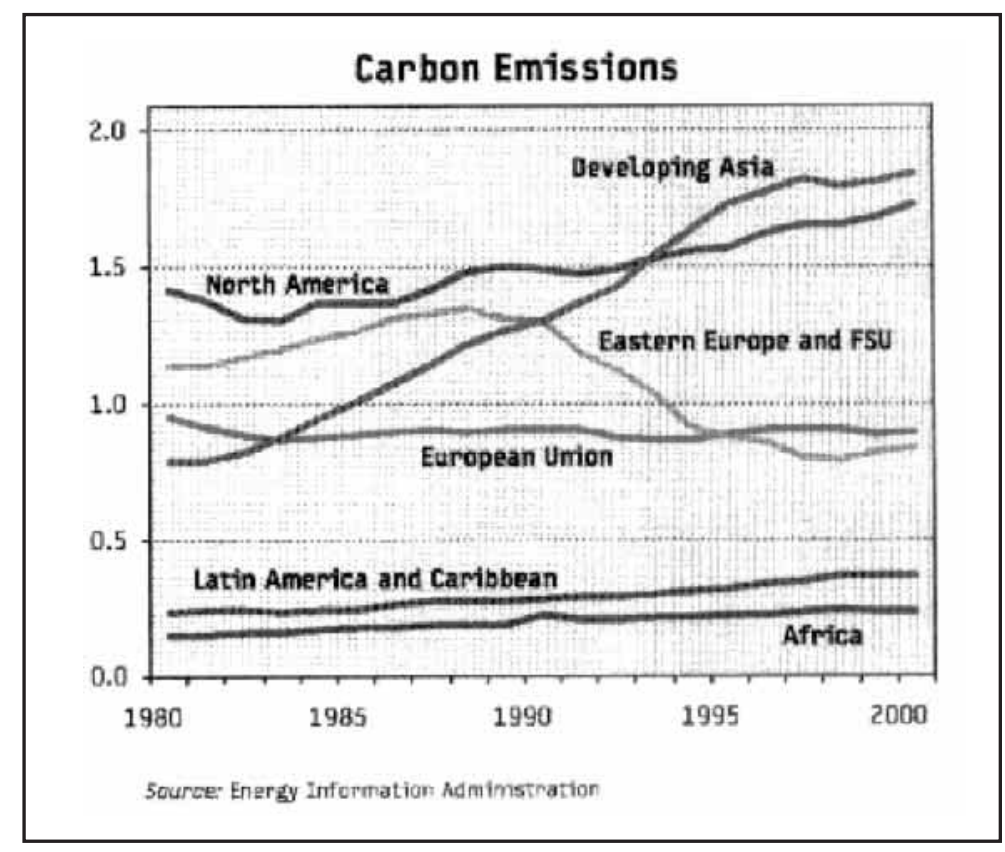

Fig. 1. Emisiones Por Región. Fuente: Cumbre Johannesburg (2002)

\section{2.- Efectos en la Sociedad Humana}

Distintos organismos Internacionales como la ONU y el Grupo Intergubernamental de Expertos sobre el Cambio Climático (IPCC, 2001), han señalado que dentro del los efectos principales del calentamiento global se encuentra el aumento del nivel del mar. Al existir un aumento de la temperatura en todo el planeta, este tiene una gran influencia en los casquetes polares, enormes masas de hielo milenario de una $u$ otra forma comienzan a ser impactadas por esta dinámica calórica.
En Chile hay varias evidencias al respecto. El estudio de los expertos de nuestros glaciares localizados en la zona de la Patagonia en un periodo de más de 50 años muestra un significativo retroceso de los hielos. Por otro lado continuamente se informa del desprendimiento de grandes masas de hielo que caen al mar, cuyo tamaño no deja de sorprender. En uno de esos casos un gran bloque de hielo desprendido tenía el tamaño de la isla de Chiloé, lo que en su momento significó además un peligro de colisión. Posteriormente dicha "ciudad de hielo" se derri- 
tió sin mayor análisis por parte de la población. No es factible todavía decir que estos fenómenos deben ser explicados por el calentamiento global, pero desgraciadamente tampoco se puede afirmar lo contrario.

Según el IPCC (2001) sería evidente el aumento del Nivel del Mar. Hay cálculos más optimistas y otros más dramáticos, pero una apreciación moderada estima que el mar aumentaría en 88 centímetros para el 2100.

Este aumento afectaría a muchos lugares del mundo, las costas de varios países, y principalmente países insulares Rosas, M. (2001) señala que prácticamente desaparecerían bajo el agua millones de personas de países en desarrollo. Poblaciones afectadas en China, Bangladesh y Egipto quedarían sin hogar.

El Calentamiento global trae consigo además cambios climáticos que conllevan fenómenos como ciclones, huracanes, sequías, lluvias y enormes inundaciones. Esto, como es de esperar, tiene un lamentable impacto en perdida de vidas humanas, sin embargo el efecto es mayor todavía en tanto cuanto estos desastres inciden en los sistemas productivos, en las cosechas, en la agricultura, prolongando las consecuencias de estas turbulencias climáticas hacia la falta de alimentos, hambre, pobreza, y diferentes flagelos que se mantienen en el tiempo.

Lamentablemente los efectos producen una mayor pobreza en países que ya se encontraban en malas condiciones sociales y económicas. Estos países verían afectada en un $25 \%$ su producción de alimentos, cuando ya 450 millones de personas se encuentran mal alimentadas. En definitiva muchos países pobres, no tienen margen para mayores perdidas, y su pobreza se acrecienta.

Según algunos cálculos 65 países en desarrollo para el año 2030 habrán perdido su capacidad para producir 280 millones de toneladas de cereal. Es el caso de del Sudeste Asiático, donde viven dos tercios de la po- blación peor alimentada del mundo, la situación de India también es dramática, país que perdería 125 millones de toneladas de cereal al año (IPCC, 2001).

Las pérdidas económicas han aumentado en el mundo debido a desastres naturales provocados por cambios climáticos Los 4.000 millones de dólares anuales perdidos en la década de 1950 han quedado atrás en relación a las perdidas más recientes que según algunos cálculos ascienden a 40.000 millones de dólares en 1999.

Sin embargo aquí hay un tema de equidad y de justicia. Los países pobres o en desarrollo han contribuido poco a las causas del calentamiento global, sin embargo, muchos de ellos soportarán el peso de los cambios climáticos.

Todo el planeta se verá afectado, sin embargo algunos tendrán efecto positivo. Canadá y Rusia ahora podrán cultivar en zonas que ahora están cubiertas de hielo. Otros países beneficiados serían Finlandia, Noruega, Nueva Zelandia.

Como se puede observar los efectos del calentamiento global impactan diferenciadamente a los países del mundo. Tales situaciones dan como resultado condiciones de desarrollo carentes de sustentabilidad para todo el planeta.

\section{3.- Efectos en la Biodiversidad. Efectos en la Flora y Fauna}

El aumento de la temperatura del planeta está teniendo también efectos en la flora y fauna del planeta. Se han constatado decenas de casos, desde las regiones polares hasta los mares tropicales, de ecosistemas modificados por efecto del cambio global.

Durante el siglo XX se ha constatado un desplazamiento de especies hacia los polos y hacia las alturas. Especies vegetales 
foráneas invaden ecosistemas de los Alpes Suizos a medida que se reducen los días de heladas cada invierno.

El problema para las especies es que se deben adaptar a los cambios de su entorno o simplemente no sobreviven. No obstante, el cambio climático inducirá la extinción de muchas especies que no podrán adaptarse. Las extrapolaciones realizadas indican que ya se estarían extinguiendo entre 10.000 a 50.000 especies al año (Malcom y Markham, 2000).

Por otro lado hay pruebas de la expansión de enfermedades transmitidas por mosquitos en las regiones altas de Asia, del Este de África y América latina, debido a la movilidad que exige la necesidad de evitar la muerte, de los mismos insectos.

La sobrevivencia de muchas especies dependerá de su habilidad para escapar de zonas climáticas desfavorables. Esto quiere decir de la capacidad de migración hacia un territorio que ofrezca condiciones de vida más apropiadas.

Se realizó un interesante estudio sobre la velocidad de migración requerida para mantener la sobrevivencia de las especies sin ser destruida por los cambios climáticos. Malcom y Marhham (2000) señalan que el aumento de la velocidad de migración es una señal significativa y un indicador de que algo grave está pasando. Los datos indican que las especies deben salir o migrar más rápido de su territorio que en el pasado.

En este estudio se calcularon las tasas de migración requeridas para la sobrevivencia de diferentes especies, denominadas TER. Esta velocidad corresponde a la mínima necesaria, para escapar del cambio del clima. Los resultados muestran que no todas las especies pueden alcanzar dichas velocidades, como asimismo las Ters para las especies vegetales son 10 veces superiores a las que se registraron en la última era Glaciar, indica el estudio.
Se concluye que estas tasas de migración tan elevadas afectan del $17 \%$ al $21 \%$ de la superficie del mundo. Algunas especies podrán desarrollar esa velocidad, pero tasas de esa magnitud afectarán a otras no lo suficientemente rápidas, generándose extinción, Por otro lado existen regiones del mundo donde no se ha producido una selección basada en la movilidad, es decir algunas especies han evolucionado in situ y serán totalmente incapaces de emigrar.

Es interesante revisar estos temas asociados a la fauna, pero hay que constatar que en este ámbito habitualmente y prioritariamente nuestra preocupación son los problemas que afectan a la sociedad humana y la tendencia cotidiana es ver sólo como un accesorio los perjuicios que sufren otros seres vivos, sin comprender que tanto el ser humano como el resto de las especies viven en interacción permanente, e indudablemente estas consecuencias impactan a unos y por ende afectan también a los seres humanos.

\section{4.- Enfoques Ambientales:}

Si bien la causa directa del calentamiento ambiental es el efecto invernadero y a su vez este último es provocado por la sobre emisión de gases tóxicos arrojados por las sociedades humanas, bien vale ir más atrás en la secuencia de las causas e intentar llegar a las ideas de fondo, a los valores que sustentan el paradigma que actúa como soporte de este proceso.

Existen varios paradigmas, pero aquí nos concentraremos sólo en tres de ellos, que presentan o representan y aglutinan el grueso de las posiciones en torno al tema en estudio.

Para focalizar el análisis, los paradigmas que se exponen a continuación no son referidos al calentamiento global propiamente tal sino sobre el concepto de medio ambiente y desarrollo. El tema del calentamiento global está, por decirlo así, incluido en las 
miradas que se hacen sobre la naturaleza y más bien se refiere a una consecuencia de los tipos de aproximación que realiza la sociedad humana a los recursos naturales.

En primer lugar el Paradigma "Economía de Frontera", asociado fundamentalmente a la tecnología, a la modernidad y, como dice su nombre a la economía, a sido el paradigma dominante y es el que ha sustentado nuestra acción social hasta recientemente, la década del 60-70, en que comenzó masivamente a ser cuestionado (Llena, F., 2001).

Plantea que los recursos naturales son infinitos, que el planeta siempre los proveerá, que los recursos naturales son inagotables o en su defecto, la ciencia, creará los medio necesarios para obtenerlos. La ciencia, sin embargo, aquí ha sido usada fundamentalmente no para buscar recursos donde no hay sino para maximizar la obtención de recursos donde si hay.

Con esta mirada este paradigma ha promovido una actitud absolutamente ajena a la protección y conservación, concibiendo a todos los recursos por igual, no haciendo ninguna distinción entre ellos. Su propuesta final prácticamente es un no concepto, una no definición sobre el medio ambiente y su proyección en el tiempo, por lo tanto un descuido completo de su protección. Se ha calificado esta posición como antropocéntrica.

Desde una perspectiva indígena este enfoque ha sido criticado porque considera los recursos naturales, sólo como riquezas a explotar. Detrás habría una valoración del dinero y del poder. El enfoque económico que presenta está basado en el crecimiento continuo, realidad imposible de mantenerse, no es durable. Se ha supuesto que el agua y el aire, son elementos imperecederos Ha privilegiado un estilo de vida desarrollado en espacios artificiales, ciudad, lejos del medio natural, perdiendo con ello su capacidad de comprensión.
El segundo paradigma, el cual hoy día tiene más difusión y que es la contrapropuesta del anterior, es el Desarrollo Sustentable o Ecodesarrollo. Plantea principalmente el tema de la sustentabilidad, en el sentido que la explotación de los recursos naturales no debe ser explotada hasta su agotamiento sino en base a un plan moderado que permita mantenerlos en el tiempo. Esto a través de estímulos a su reproducción. Hace la distinción entre recursos renovables y no renovables. Los primeros como el agua, los bosques, etc., deben ser ocupados pero al mismo tiempo reproducidos $y$ preservados de modo de evitar su extinción como asimismo favorecer su prolongación en el futuro. Los recursos no renovables, como los minerales, el petróleo derivado de yacimientos fósiles, el gas, etc. deben ser gastados con absoluta restricción, y buscar urgentemente fuentes alternativas de uso de modo de reemplazarlos cuando se agoten. Aquí es el caso de usar otras fuentes energéticas como la energía eólica o la energía solar, (Llena, F., 2001).

El Tercer paradigma es el llamado Ecología Profunda (Naess, 1997; Merchant, 1995). Este enfoque es radicalmente opuesto al primero y va más lejos que el segundo. Critica los dos primeros señalando que de cierta manera son la misma cosa, aunque de manera diferenciada pero que ambos dos no llegan a plantear el problema de fondo como tampoco a proponer soluciones definitivas. En esencia este paradigma plantea un rechazo de plano al crecimiento económico, dado que siempre será depredador, agrega que lo fundamental es el respeto a la vida, y ello es tan importante para el ser humano como para los animales y las plantas por igual. Se señala que no existe una jerarquía, y por lo mismo los seres vivos tienen los mismos derechos. Señala que lo que ha ocurrido es un abuso por parte de una especie por sobre otras, donde la superioridad del ser humano incluso no sólo está llevando a la destrucción y muerte de otras especies, recordemos las cifras entregadas más arriba, sino que además está desestabilizando el planeta en su conjunto. 
Como se observa, existe una oposición entre estos enfoques, particularmente en el de Economía de Frontera y el de Ecología

Profunda, indicado en la siguiente tabla.

\section{Paradigmas Contrapuestos}

\begin{tabular}{|l|l|}
\hline \multicolumn{1}{|c|}{ Economía de frontera } & \multicolumn{1}{c|}{ Ecología profunda } \\
\hline Dominación sobre la Naturaleza & En armonía con la Naturaleza \\
\hline $\begin{array}{l}\text { Ambiente natural, recursos para los } \\
\text { humanos }\end{array}$ & $\begin{array}{l}\text { Toda la naturaleza tiene un valor } \\
\text { intrínseco }\end{array}$ \\
\hline $\begin{array}{l}\text { Crecimiento material y económico } \\
\text { creciente para la población }\end{array}$ & Necesidades simples \\
\hline $\begin{array}{l}\text { Creencias en recursos y reservas } \\
\text { amplias, ilimitadas }\end{array}$ & Reservas terrestres limitadas \\
\hline Progreso y soluciones de alta tecnología & Tecnología apropiada, ciencia no dominante \\
\hline Consumismo & $\begin{array}{l}\text { Vivir con lo que es suficiente/ } \\
\text { reciclaje / eficiencia. }\end{array}$ \\
\hline Comundad centralizada/ Naciones & Tradiciones minoritarias/ bioregiones \\
\hline
\end{tabular}

Fuente: Gallardo, M.(2003)

\section{5.- Hacia un Paradigma Ambiental en la Psicología Comunitaria}

Al hacer referencia a la Psicología Comunitaria Ambiental, estamos relacionando dos conceptos: lo ambiental y lo comunitario. Indudablemente esto es una pretensión, no es un hecho, pero por un lado existen antecedente que emergen de las mismas definiciones de comunidad que aluden a su carácter territorial (Sánchez Vidal 1991). Además existe una historia fuertemente asociada entre las comunidades y el respeto al medio ambiente, especialmente en comunidades tradicionales como son las indígenas y las campesinas. Sin embargo, todo ello no nos permite concluir que la sola presencia de una comunidad trae aparejado una conducta proactiva hacia el medio ambiente.

Justamente en este punto es donde corresponde decir que la Psicología Comunitaria tiene aquí la pretensión de asociar dichos conceptos. Y en esta línea esto se transforma en un propósito o en un objetivo. Si aceptamos la propuesta hecha por Berger y Luckman (1968) sobre la construcción social de la realidad, es claro que una relación entre comunidad y ambiente es también una construcción. $\mathrm{Y}$ en este proceso las comunidades tradicionales si tendrían camino andado, tanto en su historia y como en sus proyectos, la variable medio ambiental ha estado presente en su dinámica. Hay aproximaciones medioambientales instaladas en la memoria colectiva de las comunidades en general y, además actualmente existen comunidades indígenas y otras que desarrollan sus actividades considerando su inserción en el medio ambiente, de modo sustentable particularmente en continentes como Latinoamérica. (Hiraoka, 2001)

Entonces en síntesis ¿qué tenemos?, algunas predisposiciones comunitarias hacia lo 
ambiental, también algunos recursos presentes en el acervo cultural de las comunidades, pero no tenemos de ningún modo un comportamiento proambiental asegurado. Surge así dentro de la Psicología Comunitaria el objetivo de promover e insertar en las comunidades un comportamiento de protección y desarrollo del medio ambiente en el contexto de una interacción mutua.

Y en esta tarea la Psicología Comunitaria se hace consonante con el conjunto de disciplinas, como la ecología, la biología, las ciencias sociales y otras, que hoy día iniciado el nuevo milenio, plantean y ven como priori- tario y además como urgente acrecentar la relación entre el ser humano y su entorno ambiental.

A continuación se señalan algunas líneas de trabajo en este sentido.

El concepto de Desarrollo Sustentable es definido como "aquel que satisface las necesidades de las generaciones actuales, sin comprometer la capacidad y los recursos de las futuras generaciones para satisfacer las suyas" (Brudtland, 1987) y es concebido como la relación entre tres pilares esenciales. Por un lado un pilar económico, por otro lado un pilar ambiental y por otro un pilar social.

\section{Desarrollo Sustentable Como Sistema}

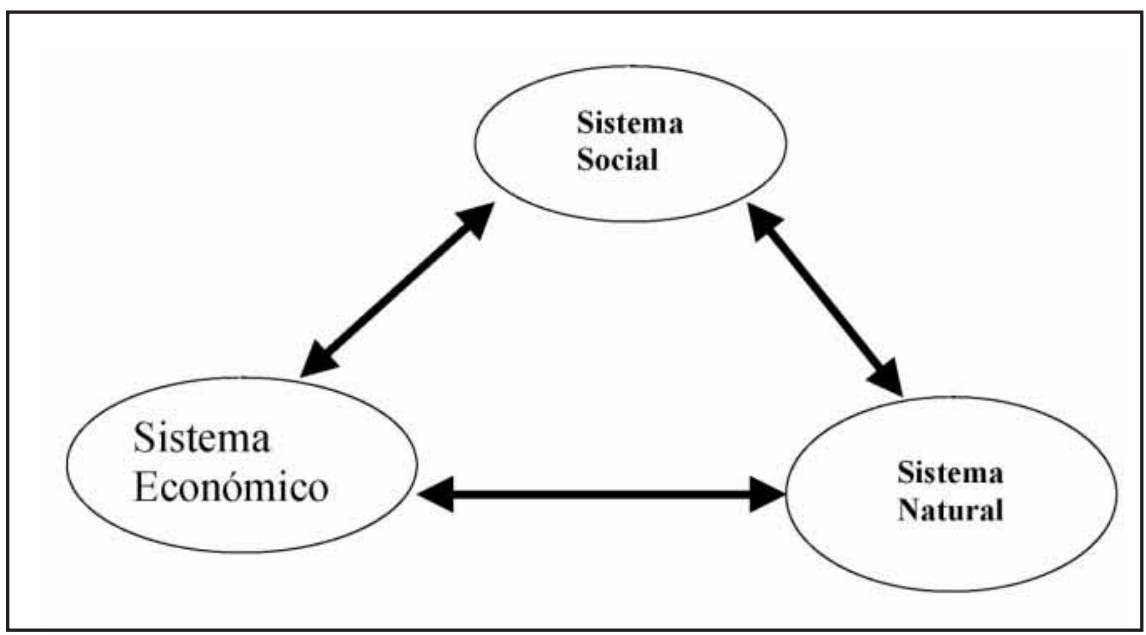

Fuente: Elaboración Propia a partir de antecedentes de Rascio, A. (1998)

Entendido el concepto de Desarrollo Sustentable como sistema, permite articular mejor las variables en juego y especialmente las interdependencias. Es clara la relación entre el Sistema Económico y el Sistema Natural, la relación aquí es histórica, aunque desgraciadamente limitada, dada la supremacía de lo económico por sobre el sistema natural.

Sin embargo el sistema que siempre ha quedado al margen ha sido el Sistema Social. Y con ello la emergencia de distorsiones o mejor dicho la aparición de calamidades sociales. Un ejemplo es la migración campociudad, producto del deterioro de los recursos naturales en el campo, lo que deriva en la instalación de los migrantes en la ciudad, pero en condiciones deplorables, generando poblaciones marginales en toda América Latina, caraterizadas por la pobreza, y la baja calidad de vida (Rozas, 2001).

Parece en consecuencia relevante complementar la definición de Desarrollo Susten- 
table con otros aspectos propios de la realidad latinoamericana. A continuación se señalan algunos.

Existen diferentes modelos de desarrollo. El modelo clásico es el modelo occidental, a través de la ciencia y la tecnología, busca pasar de la agricultura a la industria y luego a producción de servicios. Todo ello con un gran consumo de recursos naturales. Es el modelo señalado más arriba llamado economía de frontera.

Si en nuestro país y en otros países de América Latina seguimos ese modelo agotaremos nuestros recursos, dado que el nivel de consumo presente en dicho modelo sobrepasa la carga soportable por el territorio de América Latina.

Por lo mismo el Modelo de Desarrollo en América Latina debe ser diferente, debe tener otras orientaciones y combinaciones.

Un ejemplo interesante, aunque parcial, es el modelo seguido en un país Africano llamado Gabón, el cual tomó la decisión de desviar toda su economía de explotación de recursos naturales, especialmente madera y minerales hacia la industria del turismo. El turismo está basado en la oferta de 12 Parques Nacionales que presentan una gran diversidad animal y vegetal, constituyéndose un verdadero tesoro creado por la naturaleza, ahora protegido por su gobierno y disponible para la investigación y comprensión de esa realidad por parte de miles de potenciales visitantes del mundo (Quammen, D., 2003).

Este posible modelo de desarrollo latinoamericano no está creado, tal vez sólo hay lineamientos, pero es parte de nuestro problema y de nuestra tarea. Sin embargo aquí proponemos algunos elementos que debiera incluir este nuevo paradigma.

Por un lado considerar que América Latina es un continente con un fuerte componente indígena y por otro tomar en cuenta que vive en el continente un gran porcentaje, por no decir una mayoría de poblaciones de carácter popular expresada en campesinos, pecadores, mineros, obreros, etc.

Al respecto Bernardo Subercaseaux (2002) señala que en algunos países la población indígena, no mestiza, alcanza elevados porcentajes como el caso de Guatemala 66\%, Perú $40 \%$, Ecuador $40 \%$, Bolívia $63 \%$. Todos los países de América Latina registran población indígena, salvo Uruguay. De modo que en total dicha población bordea en el continente 41 millones de habitantes y está conformada por 400 grupos étnicos diferentes.

Esta fuerte presencia de comunidades indígenas y populares, permite señalar dos situaciones. En un sentido cuando se habla de desarrollo sustentable también es fundamental considerar la sustentabilidad social. Es decir, de que manera el desarrollo de la sociedad integra la cultura de estos pueblos y sectores, como asimismo sus aspectos productivos y sus territorios. Además como fortalece y hace más eficiente la utilización de los recursos naturales sin contribuir a su depredación.

Se entiende sustentabilidad social como el proceso de asegurar la inclusión de todos los grupos de población en la sociedad, a través de garantizar el acceso a la vivienda, el derecho al trabajo, a la calidad de vida, el desarrollo de su cultura, y otras necesidades básicas, en adecuada combinación y equilibrio con el desarrollo económico, el ambiente y la relación ciudad-región.

Un segundo punto aquí es un tema relacionado al capital social y cultural presentes en las comunidades indígenas y populares. Es decir existen recursos sociales que están imbricados a recursos naturales y que deben ser protegidos de modo de poder contar con ellos en el desarrollo de la región.

Es el caso de las tecnologías apropiadas, particularmente en nuestro ámbito latino- 
americano, existe gran riqueza en recursos tecnológicos asociados a dichas culturas. La particularidad de estas tecnologías es que están adaptadas al territorio donde vive la población, en general no son depredadoras del medio ambiente, son muy simples y de fácil comprensión, por lo tanto están al alcance de los usuarios y tienen un rendimiento ajustado a las necesidades de la población. Es decir cumplen requisitos de sustentabilidad, por cuanto hay un balance, un equilibrio entre las necesidades de la población, y las posibilidades de explotar los recursos naturales sin agotarlos.

Por otro lado las riquezas naturales en América Latina son significativas. Estas riquezas son una enorme fuente de conocimiento sobre el funcionamiento ecológico del continente, el caso del Amazona. Existen ya innumerables estudios que indican que los hábitats nutridos de especies cuentan con gran diversidad genética. (Hiraoka, 2001; Quammen, D., 2003). Esto es contar con un mundo de seres que alojan en sí capacidades no conocidas todavía para enfrentar la vida, potencialidades de sobrevivencia, potencialidades de adaptación, potencialidades de cambio, de relación, de articulación.

Al favorecer esta diversidad la sociedad latinoamericana tiene mucho que ganar, no sólo en cuanto a productos alimenticios y de construcción de su hábitat sino también en calidad de vida. Esto es muy relevante, por cuanto implica salud física y mental en armonía con una salud ambiental.

En este contexto la construcción de un paradigma asociado a la psicología comunitaria, debiera estar vinculado a los siguientes temas.

Las líneas temáticas de incorporación al trabajo de la Psicología Comunitaria Ambiental que se proponen son:

- Mayor aproximación al mundo Natural (Contacto con la naturaleza y animales)
- Estilos de vida: Consumo Reducido

- Sustentabilidad Social

- Interculturalidad: Incorporación de las miradas del mundo étnico-indígena

- Variables Psico Comunitarios: Identidad y Organización Comunitaria; Comportamiento colectivo

En relación a todos estos aspectos tal vez uno de los más relevantes es el de mayor contacto con el mundo natural. La sociedad humana actual nos ha empujado principalmente a la vida en las ciudades, las cuales nos alejan del mundo natural y nos ofrecen fundamentalmente condiciones artificiales de vida. Se nos aleja de la comprensión de los mecanismos de generación de la vida.. La artificialidad nos impide observar las relaciones, el funcionamiento sistémico de las especies, como así mismo los peligros de extinción y las necesidades del mundo natural. Y lo más delicado y grave es la generación de una autoimagen de seres humanos que viven independiente de estos fenómenos ambientales, más aún se genera la idea que ese mundo es sólo un accesorio, una bodega, un granero para satisfacer nuestras necesidades.

Es indispensable entonces, generar o modificar la imagen en la cual se aprecie la estrecha relación e incluso de dependencia de lo humano y dicho mundo.

Esto nos llevaría positivamente a cambiar nuestro estilo de vida, especialmente en relación a la reducción del consumo. Parece contradictorio, incluso, preocuparse del consumo dado que habitualmente los países para encaminarse al desarrollo estimulan a su población a acrecentar el consumo, sin embargo su aumento nos lleva a sobreexplotar los recursos naturales y caer posteriormente en pobreza. Disminuir el consumo pasa por entender y comprender que no se trata de no consumir sino consumir lo que se necesita 
como también de consumir de manera sustentable, dejando constantemente abierta la puerta a la reproducción de los productos de consumo y no llegar a agotarlos.

Por otro lado, el respeto a otros grupos humanos es también cuidado por sus formas de producción, por su territorio, por su lugar de vida. Esta línea nos encamina positivamente hacia la interculturalidad, la posibilidad de encuentro y enriquecimiento mutuo entre diferentes culturas y grupos. Variadas culturas disponen de interesantes aproximaciones a una mejor relación con el medio ambiente. Abrir la puerta al intercambio permitirá comprender aspectos del mundo natural con mayor profundidad y a su vez introducir tecnologías más apropiadas. El encuentro con otras culturas siempre será provechoso y contribuirá a generar mayores equilibrios.

Todo lo señalado en definitiva decanta en la necesidad de lo comunitario. Es decir en la urgencia de generar un comportamiento colectivo y no individualista respecto de estos temas. La disminución del consumo, la sustentabilidad social y la interculturalidad requieren de un cambio colectivo, de un cambio en el comportamiento de muchas personas. Y como bien sabemos en psicología, una conducta observable para que se mantenga en el tiempo debe estar asociada a cambios en la conducta cognitiva, también emocional y particularmente debe ser una conducta social, es decir representativa de un colectivo mayor.

\section{6.- Lineas de Intervención en Psicología Comunitaria Ambiental}

Las líneas de intervención de la Psicología Comunitaria en el campo ambiental y en relación al calentamiento global son variadas. Por un lado está la educación ambiental en el ámbito formal e informal, en la educación de adultos, también está la interven- ción comunitaria en proyectos ambientales que buscan cambios a través del fortalecimiento comunitario y la participación.

Sin embargo los mayores éxitos en intervención están relacionados al ámbito de lo productivo, al consumo de energía, a la planificación territorial. Esto último es de gran relevancia por cuanto permite un enfrentamiento más general respecto de lo que puede hacerse en todo un territorio, tomando en cuenta sus recursos, sus líneas de producción, sus proyectos de desarrollo, los recursos humanos, la cultura, y el capital social.

A continuación vamos a exponer dos experiencias que pueden dar cuenta de aspectos interesantes asociados a la Psicología Comunitaria Ambiental.

\section{1.- Experiencia Aprovechamiento Energía Solar en Pichasca}

Este proyecto fue realizado en las Localidades de Pichasca, Fundina, San Pedro, Caracoles, distantes aproximadamente a $50 \mathrm{Km}$. de la ciudad de Ovalle, Provincia del Limarí en la IV Región, denominado "Organizaciones de Base contra la desertificación Comunal". (Maya \& Tognola, 2003)

En esta zona existe una alta depredación de los recursos naturales (árboles, arbustos y raíces) debido a la sequía del lugar. Producto del inadecuado manejo del ganado caprino y la alta tasa de explotación del recurso leña, profundizando la situación de desertificación en esta Comuna, agravando la pobreza de sus habitantes. Estas localidades corresponden a asentamientos históricos de pastores-campesinos pobres cuya sobrevivencia peligra al tener un escaso acceso a tecnología adecuada.

El proyecto desarrollado por el Canelo de Nos, ONG, en conjunto con la comunidad, propuso un manejo controlado del recurso leña a través de la forestación de áreas co- 
munes con especies nativas de la especie Algarrobo, en combinación con la introducción de receptores de energía solar para la optimización de hornos de leña y hornos domésticos, junto a un manejo más adecuado del ganado Caprino.

La metodología se basó en la participación asociativa. Se trabajó además en función de fortalecer las redes. Particularmente la red local de capacitación (Liceo Agrícola, Programa Servicio País, Programa de educación Rural Ambiental Integral del Dpto. de educación del Municipio de Río Hurtado).

También en dirección a promover la participación de las organizaciones territoriales a partir de la Junta de Vecinos de Pichasca y la asesoría técnica del Canelo de Nos y de la Corporación Nacional Forestal (Conaf).

Los resultados fueron muy significativos. Por un lado los habitantes adquirieron competencias y capacidades que les permitieron valorar, tomar conciencia e intervenir técnicamente y de modo sustentable en el entorno que les rodea.

Avanzaron en recuperar el valor nutricional de los suelos aportando además mayor capacidad de retención y distribución de aguas, con mayor tecnología apropiada de riego y uso más eficiente del agua. Comenzaron a realizar un consumo consciente y con perspectivas de futuro del forraje y la madera.

Ahorraron en el consumo energético a través de la optimización de artefactos a leña e introducción de artefactos de recepción de energía solar. Incrementaron hábitos de higiene al trabajar con energías limpias (energía Solar) y aumentaron su el tiempo disponible para otras labores.

Incrementaron sus posibilidades de arraigo y permanencia evitando acrecentar el flujo migratorio. Fortalecieron sus organizaciones y sus capacidades de empoderamiento para la intervención en la política y en planeamiento comunal.

\section{2.- Gestión Territorial Palancas de Cambio}

Las propuestas que se señalan a continuación son producto de una investigación sobre la intersectorialidad en Programas que trabajan con Pobreza realizada en la VI y VIII Regiones (Rozas 2001, 2002).

Para orientar un trabajo de carácter ambiental desde la Psicología Comunitaria se propone utilizar el Modelo de Macro-Redes Intersectoriales, (Rozas, 2002) el cual tiene tres elementos: un enfoque territorial, la metodología de Diagnóstico de Dinámicas, y el trabajo en redes, aspectos que se explican a continuación.

\section{Acerca del Enfoque Territorial}

Este enfoque asume una perspectiva topológica para la comprensión de las problemáticas sociales, tomadas a partir de su localización y arraigo en una zona geográfica delimitable, además de una mirada igualmente tópica de las posibles soluciones y estrategias de enfrentamiento. Pretende trascender el enfoque estático y parcial que se deriva de revisar dichos fenómenos sólo a partir del acopio de datos estadísticos, que permiten una mirada analítica de la situación pero no su síntesis. Mediante este enfoque se abre la posibilidad de poner en relación los fenómenos de desarrollo y medio ambiente de una localidad o zona geográfica con los ocurrentes en otras localidades o zonas, sean éstas contiguas o bien remotas.

Una vez que se ha desplegado el conjunto de elementos necesarios que identifican el espacio territorial donde se está trabajando se sugiere realizar un diagnóstico de dinámicas.

\section{Diagnóstico de Dinámicas}

El diagnóstico de dinámicas es un método que tiene como objetivo facilitar la identificación de procesos (sistemas) relacionados con 
el desarrollo y la selección de "palancas" o estrategias de intervención que sean pertinentes para superar los problemas presentes.

El diagnóstico de dinámicas contempla cuatro pasos fundamentales. El primero es la caracterización de los grupos de interés, de las condiciones del territorio y de su entorno. La idea aquí es ir diferenciando las comunas, localidad o subterritorios según sus distintos "perfiles", ya sea en términos de nivel educacional, actividad laboral, recursos productivos y accesibilidad vial.

El segundo paso del diagnóstico de dinámicas consiste en identificar, analizar y establecer relaciones entre los diversos problemas, aspectos instituciones que afectaban a los grupos de interés poblaciones en relación a la temática ambiental, en función de buscar el o los mecanismos de reproducción de los problemas allí presentes.

El tercer paso del diagnóstico de dinámicas consiste en buscar un conjunto de objetivos posibles de alcanzar y que permita revertir los problemas antes identificados. Se considera aquellos problemas posibles de transformar para prevenir la reproducción de los problemas, aprovechando los recursos de su entorno y su capital social.

El cuarto y último paso y como complemento de lo anterior, el análisis de sistemas y estrategias de intervención, es decir, la identificación de procesos donde existiesen estrategias claves o "palancas" que permitieran generar desarrollo en la comuna o en la región. Esto implica formular una propuesta de intervención con un carácter "estratégico" en tanto fuese capaz de producir efectos positivos en aquellos procesos que se relacionaran con la mantención y reproducción de los problemas medioambientales

\section{TRABAJO EN RED:}

La constitución de redes permite tomar conciencia colectiva de los problemas y ge- nerar visiones homogéneas sobre los mismos. Si esto ocurre, se cuenta con un gran recurso social, pues así en el momento de la intervención existe una misma imagen respecto de cuáles son las necesidades y qué se debe hacer para resolverlas. Aquí nos referimos a la urgente necesidad de diagnósticos comunes; sin lugar a dudas éste es un nivel difícil de lograr, pero precisamente por ello es que se requiere el trabajo en red. La red es un grupo amplio de distintas instituciones y también de distintas miradas, y uno de sus elementos es proporcionar un espacio de negociación: es un momento de acuerdos y de conflictos, sin embargo ello en tanto proceso lleva a desarrollar una visión integral de la realidad social y a incorporar distintas apreciaciones; se enriquecen el diagnóstico y las soluciones.

La red es un espacio de aprovechamiento de recursos, en el que cada participante incorpora propuestas, capacidades profesionales y aportes financieros. El tema crucial aquí es que dichos recursos no se ocupen en forma desarticulada, sino con cierta coordinación. Para que ello sea efectivo, debe haber una conducción, la cual debe venir del territorio; es decir, son los requerimientos de ese espacio socio-productivo los que deben ir señalando las prioridades. La red, al estar consciente de ello, podrá así ensamblarse adecuadamente para actuar en conjunto, pero no todos al mismo tiempo sino en continuidad, de manera secuenciada, con complementariedad e integralidad.

\section{Actividades Fundamentales de la Red:}

Estas son: diagnóstico, planificación, intervención y evaluación. Un diagnóstico compartido entre los miembros de la red permitirá optar por un tipo determinado de intervención, articular adecuadamente los recursos institucionales y al conocer la evaluación de impacto territorial, la red favorecerá una mejor reorientación de todo el proceso. En este caso es fundamental contar con apoyo informático 
de manera de ir conformando una memoria territorial de diagnósticos. Por otro lado, resulta claro que los diagnósticos deben ser dinámicos, transversales y longitudinales. En el caso de la intervención, debe orientarse preferentemente a la acción en puntos estratégicos y palancas de desarrollo. En la planificación, se debe favorecer la planificación situacional por sobre la planificación normativa; y respecto de la evaluación, privilegiar sobre todo la evaluación de impacto.
Un ejemplo visual de una parte de esta propuesta, es el Diagnóstico realizado en la VIII Región en torno la vulnerabilidad Socio territorial. No incorpora el tema ambiental, pero muestra una línea de acción para ese efecto. La vulnerabilidad Socio territorial es un indicador compuesto que mide las siguientes variables: Nivel de Pobreza, Nivel de Enseñanza Media, Nivel de Jefatura femenina, Grado de Ruralidad, Cesantía, Nivel de Servicios y Tasa de Crecimiento.

\section{Mapa de Vulnerabilidad VIII Región -chile}

\section{Vulnerabilidad Socio-Territorial en la VIII Región del Bío Bío, 2000}

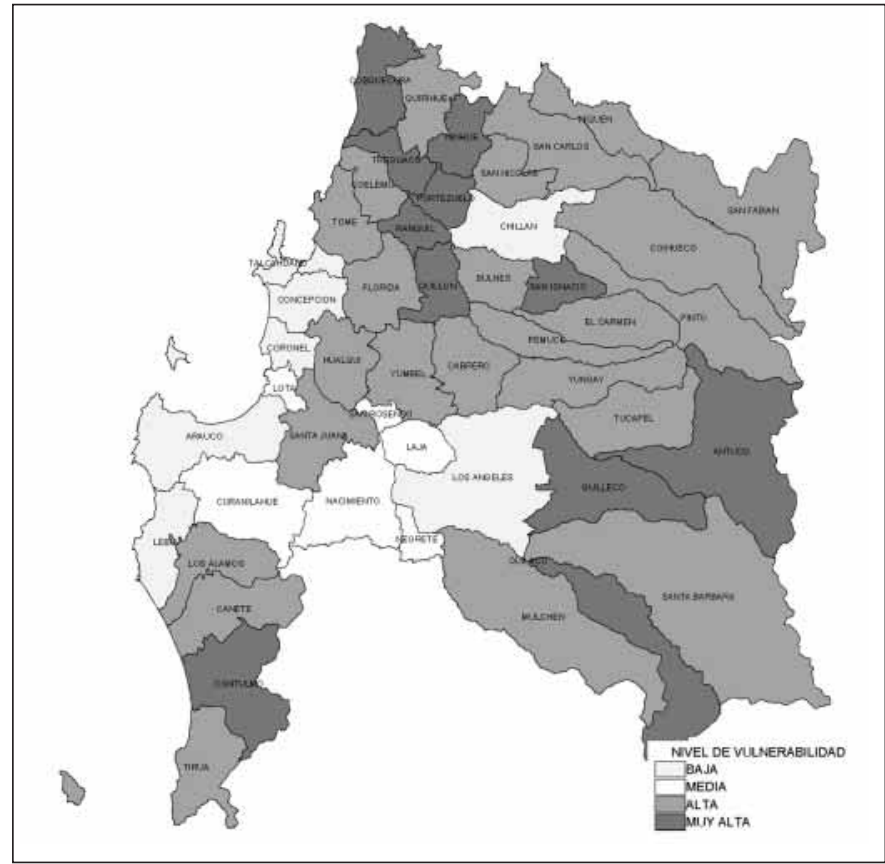

Fuente: Elaboración propia a partir del Censo de Población y Vivienda de 1992 (INE) y la Encuesta CASEN del 2000 (MIDEPLAN).

\section{7.- Reflexiones Finales}

Como se ha expuesto más arriba, el Calentamiento Global no es un tema menor, tiene graves consecuencias para la vida de la sociedad humana de hoy y del futuro. Y cada vez es más claro y evidente que es un fenómeno provocado por el ser humano.
Al mismo tiempo problemas de esta magnitud demuestran que el tema ambiental no es un tema exótico, aislado o independiente de nuestra vida, de nuestro comportamiento. Todo lo contrario, el modo en que el ser humano realiza su vida tiene un efecto directo. Surge con más claridad, en definitiva, la estrecha relación que tenemos los seres hu- 
manos con el medio ambiente, y que nuestras pretendidas expectativas de independizarnos del mismo a través del uso exclusivo de la ciencia, es equívoca.

Si lográsemos alejarnos y distanciarnos del entorno ambiental, al poco andar significará alejarnos de nosotros mismos. La conexión y buena interacción con el medio ambiental nos favorece, nos enriquece.

Por lo tanto lo que interesa aquí en cuanto a paradigma es la construcción de un enfoque que justamente permita por un lado comprender mejor esta relación, como por otro lado, mejorar, superar los errores del pasado y generar una sustentabilidad ambiental que, es claro, también es nuestra propia sustentabilidad.

Y cuando hacemos referencia al entorno ambiental, también y de modo fundamental es adecuado entender que allí está incluida la fauna. La interacción entre el ser humano con el ambiente debe incluir su interacción con el mundo animal. Ellos forman parte de una alianza indisoluble. Todos los estudios señalan que la relación entre animales y el ambiente, colabora positivamente en los procesos de consolidación de la vida.

El trabajo en este campo, no obstante, exige comprender que se requiere un enfoque integral. Es claro que estamos vinculados a un conjunto de variables y no con un aspecto en particular. Son diversos fenómenos multirelacionados que conforman un sistema, son cadenas de relaciones, entre el ser humano, la flora y la fauna y el ambiente atmosférico, que configuran un hábitat, o dicho en términos técnicos un ecosistema. Por lo tanto nuestra propuesta debe apuntar a que los productos obtenidos del medio ambiente que nutren y alimentan nuestra vida como sociedad deben caer bajo un concepto que permita la continuidad de existencia de estos hábitats o ecosistemas. Nuestros ciclos de consumo humanos deben estar acordes favorablemente con los ciclos de desarrollo de los ecosistemas.
Desde el punto de vista de la Psicología lo que se exige a la población es un cambio de comportamiento. Una conducta proambiental, un cambio de estilo de vida. Se trata de cambiar nuestros conceptos cognitivamente, pero también conservar y proteger el entorno, no sobrexplotar los recursos, disminuir el consumo, evitar la contaminación, como además se trata de conectar nuestras actividades equilibradamente con los procesos ambientales en curso.

La Psicología Comunitaria, cuya pretensión es incorporar lo ambiental, como otra de sus dimensiones, tiene en su acervo teórico y aplicado herramientas que pueden permitir hacer una adecuada conexión entre la actividad humana, las comunidades y la sustentabilidad ambiental necesaria.

Se trata de fortalecer un grupo humano cohesionado con un sentido de pertenencia, e identificada con valores de integración al entorno. Asímismo los cambios requieren del concurso de la población, su participación, su conciencia, su organización, su denuncia, su movilización, su compromiso, son aspectos que la Psicología Comunitaria puede contribuir.

\section{Referencias Bibliográficas}

Berger, P. \& Luckman, T. (1968). "La Construcción Social de la Realidad". Ed. Amorrortu, Buenos Aires, Argentina.

BRUdtland, G. (1987). "Nuestro Futuro Común", en: "Nociones Psicosociales para la intervención y la Gestión Ambiental". Monografía Socio Ambientales. Moreno, E. \& Pol, E. (2000) Ed. Universitaria de Barcelona.

Corral, V. (2002). Conductas Protectoras del Ambiente. Ed. Universidad de Sonara, CONACYT, México.

Cumbre Johannesburg (2002). “Global Challange Global Opportunity: Trend in Sustainable Development" en United States Energy Information Administration, www.eia.doe.gov. 
RAscio, A. (1998). "Desarrollo Humano Sustentable" En: Promoción Social Comunitaria, Diéguez, A. Et Al, Ed. Espacio, Buenos Aires, Argentina.

DuccI, M. E. (1997). “Chile, el lado oscuro de una política de vivienda exitosa". Revista EURE. Vol. XXIII, N 69, pp. 99-115, Julio, Sgto., Chile.

Duery, L. (1999). “Un desarrollo no sustentable: Mar Aral, hoy un desierto salado por mal uso del agua", Diario El Mercurio, 3 de Abril de 1999.

Hiraoka, M. \& Mora, S. (2001). “Desarrollo Sostenible en la Amazonía, ¿Mito o Realidad?" Colección Hombre y Ambiente $\mathrm{N}^{\circ}$ 63-64 Ed Abya-Yala Quito-Ecuador.

Gallardo, M. (2003). Ecología Profunda en www.mogensgallardo.com/deepeco/ecologia_ profunda.html.

GARCíA, C. (1996). Historia y Desastres en América Latina. Ed. CIESAS; La Red, Colombia.

Grupo INTERGUbERnAMENTAL DE EXPERTOS SOBRE el Cambio Climático IPCC, (2001). Cambio Climático 2001, Impactos, Adaptaciones y Vulnerabilidad. Ed. OMM, PNUMA.

Guddynas E. \& Evia G. (1993). Ecología Social. Ed. Popular. Madrid, España.

Guevara, J. (1998). Estudios de Psicología Ambiental en América Latina, Ed. UAM, México.

Guevara, J. (2003). Los Cambios Físicos y Sociales de la Vivienda popular en Latinoamerica. Ed. UPAEP, Puebla, México.

LEÓN, P. (1996). “Comunidades urbanas en Centro América: Vulnerabilidad y Desastres". Revista Desastres y Sociedad N7/ año 4. Ed. Red. México.

LlenA, F. (2001). Enfoque económico del MedioAmbiente en www.ciberconta. unizar.es/Leccion/medio11/inicio.html.
Malcolm, R. \& Markham, A. (2000). Global Warming and Terrestrial Biodiversity Decline, WWF Climate Change Campaign, Agosto en www.panda.org/climate.

Maya \& Tognola, (2003). Estudio de experiencias de Intervención en Psicología Ambiental en Chile desde una perspectiva Comunitaria. Tesis Universidad Sek, Stgo., Chile.

Merchant, C. (1995). Key concepts in critical theory, Ecology. Ed. Doubleday Publishers. USA.

Moreno, E. \& Pol, E. (1999). “Apropiación, Simbolismo del Espacio e Identidad Social", Nociones Psicosociales para la Intervención y la Gestión Ambiental, Ed. Universidad de Barcelona, España.

NAEss, A. (1997). The Sallow and the Deep,Longrange Ecological Movement: A Summary, Ed Ympäristöfilosofia Gaudeamus Books, Finnish University Press, Tampere.

Quammen, D., (2003). "Al rescate del país Africano" en Revista National Geographic Sep, Ed Televisa, México.

Rosas M. C., (Mayo, 2001). Página Uno, suplemento de Unomásuno. México.

Rozas G., (2002). “Desarrollo Social de la Ciudad Región" en Cap V Intervención Ambiental, Libro Temas Selectos de Psicología Ambiental Ed. UNAM, Fundación Unilibre, Greco, México, 2002.

Rozas, G., Martínez,V., Bolivar, D., Leiva E. \& CÉSPEDES, E. (2001). “Diagnóstico y Vulnerabilidad Socio territorial en la VI Región" Revista de Psicología, Volumen X, N¹ Dpto. de Psicología, Universidad de Chile.

Subercaseaux, B. (2002) Nación y Cultura en América Latina: Diversidad Cultural y Globalización. Ed. LOM, Stgo, Chile. 